\title{
Fibroblast Growth Factors: A Controlling Mechanism of Skin Aging
}

\author{
Rousilândia de Araújo ${ }^{a}$ Myla Lôbo $^{a}$ Kelvis Trindade ${ }^{a}$ Darizy Flávia Silva ${ }^{b}$ \\ Neila Pereira ${ }^{a}$ \\ aPharmacy College, Laboratory of Research in Medicines and Cosmetics, Federal University of Bahia, Salvador, Brazil; \\ bInstitute of Health Sciences, Department of Bioregulation, Federal University of Bahia, Salvador, Brazil
}

\section{Keywords}

Anti-aging $\cdot$ Fibroblast growth factor $\cdot$ Cell signaling

\begin{abstract}
Cutaneous aging is a complex and continuous biological process characterized by cellular and molecular alterations, with progressive reduction of the body's capacity to maintain the homeostasis, senescence, and/or apoptosis of the dermal cells. Fibroblast growth factors (FGF) have elicited studies to evaluate their role of repair and remodeling of the dermis during the skin anti-aging process, since they are regulatory proteins that mediate important signaling pathways and act on cell regeneration and repair processes. FGF acts primarily through binding to tyrosine kinase receptors through the autophosphorylation of their residues, promoting the phosphorylation of serine, threonine, and tyrosine residues of specific target proteins such as Raf-1, MAPK/Erk kinase, and extracellular signal-regulated kinase-1, which are part of the cascade of MAP kinases (mitogen-activated protein kinase). Then, FGF initiate signaling cascades inside the cell, where each kinase activates the following by phosphorylation, resulting in alterations of cellular functions. In addition, the FGF has a relevant role in anti-aging therapy because it is related to collagen and elastin synthesis activation responsible for skin resistance and elasticity, characteristics that are diminished with skin aging. Thus, the present
\end{abstract}

\section{KARGER}

() 2019 S. Karger AG, Basel

E-Mail karger@karger.com

www.karger.com/spp article aims to review several scientific studies that demonstrated the cell signaling involved with the action of FGF on skin aging.

(c) 2019 S. Karger AG, Basel

\section{Introduction}

Skin is the largest organ of the human body, protecting it against external aggressions, such as microorganisms, chemicals, and physical agents, including sunrays. Skin also plays a role in thermal regulation, water retention, and cell regeneration. This protective barrier is formed by dermal and epidermal cells, including specialized glands for the secretion of sebum and sweat, which form a special layer as a real protective mantle $[1,2]$. Despite these protective features, skin is permeable and absorbs substances that can either benefit or impair its function, such as those involved in skin aging.

Aging is a complex and continuous biological process characterized by cellular and molecular changes, with progressive reduction of the body's capacity to keep homeostasis and an increase in senescence and/or apoptosis. This process varies between individuals and from organ to organ, and skin exhibits the effects of the passage of time most evidently [3]. In addition, the use of ultraviolet radiation, excessive alcohol consumption, tobacco 
abuse, environmental pollution, and other factors can influence and accelerate this physiological process, causing premature skin aging [4].

In this context, finding mechanisms that restore the juvenile aspect of the skin is a research field of interest for the scientific community. Therefore, the cosmetic industry acts in this process, constantly seeking new compounds to prevent and attenuate skin aging [5-7].

Growth factors have become an important therapeutic option to avoid aging, because they are responsible for cell differentiation and maturation, being directly correlated with the minimizations of the topical esthetic changes resulting from age advancement $[5,6]$. Growth factor proteins are naturally secreted by cells and interact directly or are sequestered by the surrounding extracellular matrix for presentation to cell surface receptors. Events such as cell migration, survival, adhesion, proliferation, growth, and differentiation are triggered by binding of the specific growth factor receptor, which stimulates cell signal transduction pathways. These growth factor-stimulated cellular responses in greater proportions are involved in organ development, angiogenesis, and wound healing [8].

The introduction of growth factors in certain damaged sites in the body seeking to stimulate regeneration is clinically related to regenerative medicine, where researchers intend to replace or repair damaged cells, tissues, and organs to effectively restore normal function. The effects of growth factors can be verified clinically, for example, in bone grafting and wound healing in oral, maxillofacial, orthopedic, and cardiac surgery, in addition to the promising results presented regarding reduction of hair loss. Recently, the interest of growth factors applied in dermatology and plastic surgery has increased, both in fat grafting and in skin rejuvenation [9-11].

Among the existing growth factors, we highlight the fibroblast growth factor (FGF), which induces the synthesis of type 1 collagen and therefore presents a relevant role in the process of skin aging control. In the search for new anti-aging compounds, one of the strategies used for skin revitalization and wrinkle treatment is the stimulation of collagen formation. Collagen is the protein responsible for the structure, elasticity, and firmness of the skin and it is produced by cells called fibroblasts [12-14].

Considering the clinical need to seek new therapeutic options for aging, FGFs have led to studies to evaluate its role in the repair and remodeling of the dermis in the skin anti-aging process. Thus, the present review article aims to clarify the cell signaling pathways involved with the action of FGFs on skin aging.

\section{Considerations about the Skin and the Cutaneous Aging Process}

The skin undergoes constant changes, with a high capacity of repair and renovation. It is constituted of three main layers: epidermis, dermis, and hypodermis. The epidermis is the outermost layer of the skin. It stands out in the barrier function, essentially determined by one of its integral parts, the stratum corneum, where the melanocytes and keratinocytes are located. Keratinocytes synthesize keratin, the main protein structure of epidermis, as well as cytokines that act as chemical mediators that activate cellular processes. Thus, it is the epidermis that determines the depth of migration of the active substances through the skin, playing a major role as an obstacle to penetration, which will be the limiting step of this process $[15,16]$.

The dermis is the layer of skin that possesses the widest variety of cell types such as nerves, blood vessels, and lymph, surrounded by connective tissue formed by fibroblasts, collagen, and reticular and elastic fibers. Hypodermis is the innermost and deepest layer that connects the skin to deeper structures such as muscles and bones. It is composed of an adipose mantle which acts as a thermal insulation and nutritive reservoir, protecting the body from trauma and allowing the mobility of the skin with respect to adjacent structures [17].

Physiologically, aging is associated with loss of fibrous tissue, slower rate of cell turnover, and reduction of the vascular and glandular network. In addition, the barrier function that maintains cellular hydration is also impaired. Depending on genetics and environmental factors, the normal physiological functions of the skin may decrease by $50 \%$ until midlife [1].

Aging is a natural process that happens in all human beings and can be divided into two types: intrinsic and extrinsic aging. Intrinsic aging occurs with the passage of time, determined genetically, with slow tissue deterioration, hormonal status, and metabolic reactions, such as oxidative stress. In this case, skin atrophy and fine wrinkles occur because they mainly affect dermal elastic fibers, causing reticular dermatosis $[6,18]$.

Extrinsic aging is caused by external factors, such as chronic sun exposure or cigarette smoke [19]. The latter increases the intrinsic deterioration of the skin, which is related to an age-related progressive decline in the antioxidant capacity associated with increased production of reactive oxygen species from oxidative metabolism in skin cells [20].

Multiple biochemical pathways are modulated by overloading of these stressors, which may result in the 
suppression of the receptor of transforming growth factor $\beta$-II, increased expression of matrix metalloproteinases [21], increased inflammation through the nuclear kappa factor, as well as direct damage to the structural proteins of the skin that UV radiation can cause [22]. There is evidence that these processes of aging, intrinsic and extrinsic, have biological, biochemical, and molecular mechanisms that partially overlap.

Intrinsic aging affects the skin in a manner similar to other organs, because the telomeres continually lose part of their sequences, limiting their cellular replicative capacity. However, the main aging-related skin changes occur in the matrix and in fibroblast pattern expression, which remains in the stationary phase in the dermis for a long period of time, proliferating when there is stimulation, without shortening of telomeres [3].

Factors derived from fibroblasts are essential for the normal growth and differentiation of keratinocytes, which are affected by telomere loss. Therefore, the skin that underwent extrinsic aging and the one that underwent intrinsic aging present different characteristics. However, reduced life expectancy, decreased growth factor response, increased proteolytic activity, and disruption of extracellular matrix synthesis are typical changes observed in both [23]. Such processes act independent from and simultaneous with biologically distinct factors that affect the structure of the skin.

During the aging process, the proliferative and metabolic activity of fibroblasts decreases and their functions are impaired, leading to reduction of the synthesis of structural substances such as collagen, elastin, hyaluronic acid, and chondroitin. In addition, decreased levels of growth factors, reduced amount of collagen, abnormal accumulation of elastin, and reduction in the epidermal and dermal thickness, which manifest clinically as xerosis, wrinkles, sagging, blemishes, tonus loss, and all signs of skin aging, were observed during the aging process $[24,25]$. Thus, fibroblasts have an important role in preventing the formation of signs of skin aging due to the production of these basic structural substances. Collagen and elastin fibers provide, respectively, firmness and elasticity to the skin. Additionally, mucopolysaccharides, hyaluronic acid, and chondroitin provide skin hydration $[13,15,18]$.

\section{Growth Factors and Skin Aging}

The search for new compounds to prevent or mitigate the aging process has become a priority in the development of new cosmetic assets, which already have a range

Fibroblast Growth Factors as Anti-Aging Agents of options in use, such as antioxidants, retinoids, alphahydroxyacids, anti-metalloproteinases of silicon, and anti-glycans $[26,27]$.

Rozman and Bolta [28] and Fitzpatrick and Rostan [29] found that topical application of growth factors resulted in improved photoaging related to the formation of new collagen, epidermal thickening, and improved clinical appearance of the skin with visible reduction of wrinkles. Thus, the class of growth factors has aroused the interest of cosmeceuticals manufacturers as a promising anti-aging agent, due to studies that evaluated their repair role in cutaneous wounds through action on tissue regeneration, which demonstrated positive cosmetic results against skin photoaging [30], becoming a class widely investigated in the last decade as a skin anti-aging agent.

Growth factors are regulatory proteins that mediate important signaling pathways and act on cell regeneration and repair processes [30]. They act in the cell membrane level, activating a biochemical cascade that promotes the transcription of cyclin-dependent kinases in the cell nucleus, the major intracellular molecules that control the process of cell division.

Growth factors act mainly through binding to tyrosine kinase receptors (Fig. 1), which leads to autophosphorylation of the receptor and posterior phosphorylation of serine, threonine, and tyrosine residues of specific target proteins such as Raf-1, MAPK/Erk kinase (MEK) and extracellular signal-regulated kinase-1 (ERK) [13]. These enzymes initiate signaling cascades where each kinase activates the following one by phosphorylation, inducing mitosis and cell division and causing alterations of cellular functions [31].

The Raf-1, MEK, and ERK proteins belong to three families with different nomenclatures. ERK is a member of the MAPK (mitogen-activated protein kinase) family; MEK (extracellular signal-regulated kinase) is part of the MAPKK (mitogen-activated protein kinase kinase) family that phosphorylates and activates ERK-MAPK. Raf-1 is from the family of MAPKKK (mitogen-activated protein kinase kinase kinase) phosphorylase and activates MEK-MAPKK [32].

Thus, by stimulating the proliferation of cells such as fibroblasts and keratinocytes, growth factors play a major role in the skin repair process, inducing reepithelialization through the replacement of disorganized collagen and elastin structures and the reposition of the extracellular matrix in aged skin.

The use of growth factors is a new anti-aging strategy to rejuvenate and reverse the signs of photoaged skin. Un- 
Fig. 1. FGFs bind to FGFRs, inducing receptor dimerization, kinase activation, and transference of tyrosine residues from FGFR. This, in turn, leads to the fitting of adapter proteins and the consequent activation of downstream signaling pathways. The activated FGFR2 substrate (FRS2) recruits and activates the RAS-RAF-MAPK and PI3K-AKT pathways involved in cell proliferation and antiapoptotic activity, respectively. Activation of PKC and intracellular release of $\mathrm{Ca}^{2+}$ are induced by recruitment and phosphorylation of PLC $\gamma$, events that regulate cell motility.

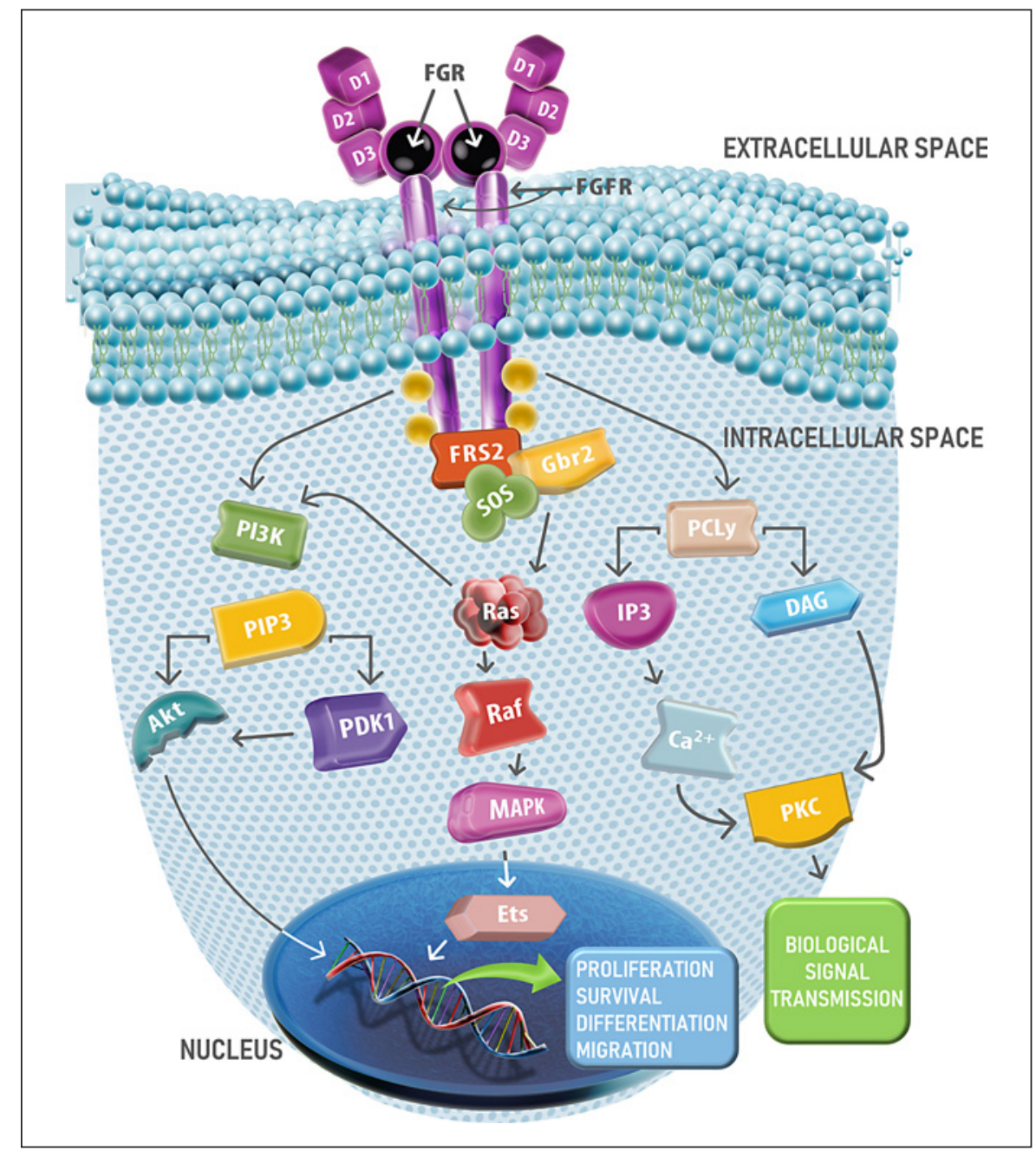

derstanding the role of growth factors in wound healing may predict their role in skin infrastructure remodeling and skin rejuvenation [33].

\section{FGF in the Control of Skin Aging}

The FGF family members increase the proliferation and activation of fibroblasts by stimulating the accumulation of collagen as well as stimulating endothelial cell division. Thus, FGFs stimulate angiogenesis, having an important function in the cell repair process $[6,13]$.

FGFs comprise a growing group of structurally related polypeptide mitogens, which includes 23 different members. In addition to the previously discovered acid FGF (aFGF, FGF-1) and basic FGF (bFGF, FGF-2), the family also includes int-2 (FGF-3). proto-oncogene products (FGF-4), FGF-5, and FGF-6, as well as keratinocyte growth factor (KGF, FGF-7), androgen-induced growth factor (AIGF, FGF-8), GAF (FGF-9), and FGF-10, among others [34].

With the aging process, fibroblasts have their activity diminished and consequently the synthesis and activity of proteins that guarantee elasticity and resistance such as elastin and collagen are also affected. Thus, in aged skin, there is a lower production of collagen by the fibroblasts and a greater action of the enzymes that degrade it. This lack of balance speeds up the aging process [12, 25].

FGF is a family that presents several forms, two of which stand out in the cell signaling process that correlates with aging: fibroblastic acid growth factor (FGFa) and basic fibroblastic growth factor (FGFb), which affect recruitment, activation, mitogenesis, migration, and differentiation of various cell types. FGFa and FGFb are secreted by multiple cell types, such as mast cells, endothelial cells, macrophages, and fibroblasts $[35,36]$. 
Most FGFs have a very broad mitogenic spectrum and perform various activities such as stimulating the proliferation of a variety of mesodermal, ectodermal, and endodermal cells and acting as neurotrophic and angiogenic factors in vivo [37]. A study by Werner [34] has shown that FGFa and FGFb stimulate the meso-, endo-, and ectodermal origin of fibroblasts as well as cell proliferation and differentiation through their mitogenic action.

FGF receptors are transmembrane tyrosine kinases with two or three immunoglobulin-like domains and a highly acidic region in the extracellular portion. FGFs bind to tyrosine kinase receptors causing its dimerization and initiating the intracellular signal transduction cascade [38]. Different members of the FGF family can bind to the four different known FGF receptors (FGFR1-FGFR4) with different affinities, and can act in a complementary way, resulting in several biological phenomena such as development [39], differentiation, migration, survival, cell division and proliferation [40], and wound healing [41].

Although the functions of FGFs are well characterized, their mechanisms of action are still not completely clear. It is known that it involves inter- and extracellular signaling pathways that may be related to the RAS-MAP kinases pathways, PI3K -AKT, PLC- $\gamma$, or STAT. Therefore, FGF cell signaling involves interactions with multiple cell signaling pathways and complex feedback mechanisms [42].

Phosphatidylinositol-3-kinase/serine-threonine kinase (PI3K-AKT) are enzymes that act at different stages of intracellular signal transduction. PI3K/Akt-mediated signal transduction may be initiated by interacting with receptor tyrosine kinases or by binding with the small G protein, Ras [43]. In this way, they present a function in regulating the proliferation, migration, survival, and cellular metabolism, as well as in gene expression, calcium flux, inflammatory response, and cytoskeletal rearrangements [44].

Phospholipase C- $\gamma$ (PLC- $\gamma$ ) is an isoform of the PLC family and its activation results in the formation of two products: inositol 1,4,5-triphosphate and diacylglycerol (DAG). The first one induces changes in cytosolic free $\mathrm{Ca}^{2+}$ concentration $\left(\mathrm{Ca}^{2+}\right)_{\mathrm{i}}$. DAG is important for the response of agents such as the growth factor, since it is responsible for activating protein kinase $\mathrm{C}$ isoforms; the formation of inositol in turn is related to the transient increase in intracellular free $\mathrm{Ca}^{2+}[45]$. The former causes a transient increase in intracellular free $\mathrm{Ca}^{2+}$, while the latter serves as a direct activator of several protein kinase $\mathrm{C}$ isoforms and thus is important for the response of agents such as growth factor.

The transcription factor family of signal transducers and transcription activators (STAT) is related to various cytokine signaling pathways, especially those that activate the gp130 membrane receptor. Through the JAK-STAT (janus-family tyrosine kinase/signal transducer and activator of transcription) signaling pathway, many cytokines and growth factors exert their biological effects. STATs form homo- and heterodimers when phosphorylated, which allows their passage into the nucleus, where they can regulate gene transcription [46].

The pharmaceutical formulations containing growth factors such as FGF has therefore become a dermo-cosmetic option for anti-aging products, as levels of growth factors decrease with the advancement of age and these in turn have functions which are important in the repair of the extracellular matrix $[4,13,20]$.

\section{FGFs Related to the Aging Process}

\section{Acid Fibroblastic Growth Factor (FGF-1, FGFa)}

The activation of FGFs promotes effects that help in the process of aging delay. Activation of FGF-1 improves skin elasticity and induces the synthesis of collagen and elastin [4, 13, 47]. Xie et al. [48] applied FGFa to rat ulcers and observed the proliferation and migration of epithelial cells and fibroblast augmentation.

FGF-1 belongs to a large family of growth factors that bind to transmembrane receptors with a cytoplasmic tyrosine kinase domain. FGF-1 is a powerful mitogen involved in the stimulation of the synthesis and proliferation of DNA from a wide variety of cell types, including mast cells, endothelial cells, macrophages, and fibroblasts. It plays important roles in various stages of development and morphogenesis, as well as in angiogenesis and wound healing processes [24]. Therefore, due to its mitogenic functions, FGF-1 has become a potential active ingredient with a wide range of uses in cosmetology directed to various processes of skin regeneration, stimulating meso-, endo-, and ectodermal cells, including fibroblasts, keratinocytes, macrophages, and endothelial cells.

One study investigated the impact of FGF-1 on skin cells and assessed the anti-aging properties of a genetically modified FGF-1 to improve its stability and resistance to protease degradation (Q40P/S47I/H93G recombinant FGF-1 [rFGF]) as an active agent in cosmetics [33]. Results showed that rFGF-1 has a strong effect on cellular proliferation of keratinocytes and fibroblasts, which was confirmed by all the tests (in vitro, ex vivo, and in vivo) with the Q40P/S47//H93G rFGF-1, suggesting a high potential for the use of this protein in anti-aging skin products and in wound healing. 
Positive effects were also obtained in the study by $\mathrm{Ha}$ and colleagues [49], who established a scalable expression system for recombinant human aFGF (rh-FGF1) using transient and a DNA replicon vector expression in Nicotiana benthamiana. The study evaluated the effect of growth factor on UVB-induced skin photoaging. The results of this study showed that rh-FGF1 not only increased cell viability but also cell proliferation. The treatment was also shown to have a significant protective effect against UVB-induced cytotoxicity, in addition to recovering the amount of procollagen lost by the radiation. Therefore, rh-FGF1 has been shown to be highly beneficial for the prevention of UVB-induced oxidative effects on skin cells, possessing a potential as a cosmetic and therapeutic agent that can be applied to prevent photoaging.

\section{Basic Fibroblastic Growth Factor (FGF-2, bFGF)}

The basic FGF (FGF-2) reduces and prevents expression lines and wrinkles through the activation of new skin cells and stimulates the proliferation of cells of mesodermal, ectodermal, and endodermal origin, mainly fibroblasts and keratinocytes. All of this suggests that FGF-2 may be important in modulation of normal processes such as angiogenesis, wound healing, and tissue repair, as well as anti-aging action $[4,13,47]$. To develop its actions, FGF-2 binds to high-affinity tyrosine kinase receptors, whose activation leads to receptor autophosphorylation and phosphorylation of other substrates, which can stimulate cellular activities such as proliferation, motility, and differentiation [50].

In order to protect FGF-2 from thermal proteolysis in aging skin and preserve its beneficial effects on the proliferation of fibroblasts, a study evaluated the activity of Hibiscus abelmoschus seed extract as a protective ingredient [51]. The results demonstrated that the extract exhibited a dose-dependent protective activity of FGF-2, which allowed the stimulation of cell turnover through this factor. In this same study, Rival et al. [51] evaluated in 60 healthy human volunteers anti-aging properties of the extract, in a double-blind placebo-controlled clinical study. Significant improvements in wrinkles, texture, elasticity, and density of the skin have been demonstrated, at least in part, due heparan sulphatelike properties of this plant and protected FGF-2 from thermal degradation. In conclusion, $H$. abelmoschus seed extract revealed anti-aging activity by protecting the FGF-2 content present in human skin, so that it can maintain its numerous functions in favor of skin rejuvenation.
In recent years, topical and injectable growth factors have emerged as an intriguing therapeutic modality that can be harnessed for esthetic and medical purposes. A group of researchers, who also studied FGFs, aimed to evaluate an in vivo method for aged skin rejuvenation through direct injection of intradermal FGF-2 [52]. The following rejuvenating effects were observed: improvement of skin smoothness, atrophied skin thickness, and improved viscoelasticity. The changes of viscoelasticity pre- and post-treatment were comparable to an age difference of more than 20 years.

\section{Keratinocyte Growth Factor (FGF-7, KGF)}

Some anti-aging products containing growth factors have already been developed. An example is the keratinocyte growth factor (KGF), which is a member of the FGF family. While most FGFs influence the proliferation and/ or differentiation of various cell types, KGF appears to act specifically on epithelial cells [34].

A study aimed to investigate the efficacy, tolerance, and use of a product containing cycloastrenol (a purified extract), antioxidant peptides, and high concentrations of three growth factors: transforming growth factor beta 1 , epidermal growth factor, and KGF. The ability of reducing the visible signs of aging, including fine lines and wrinkles, texture, pore size, elasticity, redness, hydration, and overall skin quality, was evaluated, through the observation of visible signs in photographs. The results showed that eighteen of the twenty subjects experienced significant improvement after only 2 weeks of use for all measured categories and $100 \%$ of study subjects noticed improvement in at least 3 or more of the 8 categories assessed, concluding that the product is effective for individuals who seek significant improvement in the appearance of aged skin [53].

\section{FGFs and Cosmetic Application}

According to the cited evidence about prevention of cutaneous aging induced by growth factors, pharmacological products containing different types of FGFs were identified and they are listed in Table 1.

\section{Conclusion}

The use of growth factors is getting solid in the cosmetic market as a new therapeutic option to prevent and control the aging process, due to its actions in the cellular 
Table 1. Relation of skin anti-aging cosmeceuticals composed by fibroblast growth factor (FGF)

\begin{tabular}{|c|c|c|c|}
\hline Product & Manufacturer & FGF & Other active principle \\
\hline TNS Recovery Complex ${ }^{\circledR}$ & Skin Medica & bFGF & Isolated \\
\hline D Cosmeceutical Eye Cream ${ }^{\circledR}$ & Dermaheal & aFGF, bFGF & EGF, IGF, peptides \\
\hline Doctor Duve $^{\circledR}$ & Dermaheal & bFGF, aFGF & IGF, VEFG, peptides \\
\hline Firm-X $\mathrm{X}^{\circledR}$ & Peter Thomas Roth & bFGF & TGF- $\beta$ \\
\hline Derma Heal SR ${ }^{\circledR}$ & Dermaheal & bFGF & IGF, EGF \\
\hline Peau Magnifique Youth Recruit ${ }^{\circledR}$ & Révive & aFGF & EGF \\
\hline Re Luma Skin Illuminating Anti-Aging Serum ${ }^{\circledR}$ & Re Luma & FGF & PDGF, VEGF, IGF-3, TGF- $\beta$ \\
\hline Serum Cellular Repair Gene Therapy ${ }^{\circledR}$ & E'shee & aFGF & Isolated \\
\hline Alaur Rederma Cellular Energizing Serum ${ }^{\circledR}$ & Alaur & $\mathrm{aFGF}$ & Isolated \\
\hline Skin Factor ${ }^{\circledR}$ & Buona Vita & bFGF & EGF, IGF, VEGF \\
\hline Ionto Bio Skin ${ }^{\circledR}$ & Buona Vita & bFGF & Isolated \\
\hline
\end{tabular}

EGF, epidermal growth factor; IGF, insulin growth factor; TGF- $\beta$, transforming growth factor $\beta$; VEFG, vascular endothelial growth factor; PDGF, growth factor derived from platelets. * Product is being tested [Zeranska et al., 2016].

differentiation process. FGF plays a relevant role in antiaging therapy because it is related to the induction of collagen and elastin synthesis responsible for skin elasticity and resistance, characteristics that are diminished with skin aging, both intrinsic and extrinsic. However, further studies are needed to fully understand the mechanisms of the action of growth factors, especially the FGF. A better comprehension of how these substances work would allow the development of more effective and safer pharmacological product with an esthetic aim.

\section{Disclosure Statement}

The authors have no conflicts of interest to declare.

\section{Author Contributions}

Rousilândia de Araújo, Myla Lôbo, and Kelvis Trindade reviewed the topics in the databases and wrote the article. Darizy Flávia Silva suggested the structure of the article and made corrections. Neila Pereira corrected the article.

\section{References}

1 WHO. Physiology of normal skin. In: Guidelines on Hand Hygiene in Health Care: First Global Patient Safety Challenge Clean Care Is Safer Care. Geneva: World Health Organization; 2009. [Cited 2018 Mar 17]. Available from: https://www.ncbi.nlm.nih.gov/books/ NBK144027.

2 Venus M, Waterman J, McNab I. Basic physiology of the Skin. Surgery. 2011;29(10):4714.

3 DiLoreto R, Murphy CT. The cell biology of aging. Mol Biol Cell. 2015 Dec;26(25):452431.

4 Fisher GJ, Kang S, Varani J, Bata-Csorgo Z, Wan Y, Datta S, et al. Mechanisms of photoaging and chronological skin aging. Arch Dermatol. 2002 Nov;138(11): 1462-70.

5 Maddaluno L, Urwyler C, Werner S. Fibroblast growth factors: key players in regenera- tion and tissue repair. Development. 2017 Nov;144(22):4047-60.

6 Mine S, Fortunel NO, Pageon H, Asselineau D. Aging alters functionally human dermal papillary fibroblasts but not reticular fibroblasts: a new view of skin morphogenesis and aging. PLoS One. 2008; 3(12):e4066.

7 Ito K, Yamada R, Matsumoto N, Imamura T. Evaluation of fibroblast growth factor activity exerted by placental extract used as a cosmetic ingredient. J Cosmet Dermatol. 2018 Oct; 17(5):821-29.

8 Mitchell AC, Briquez PS, Hubbell JA, Cochran JR. Engineering growth factors for regenerative medicine applications. Acta Biomater. 2016 Jan;30:1-12.

9 Pocaterra A, Caruso S, Bernardi S, Scagnoli L, Continenza MA, Gatto R. Effectiveness of platelet-rich plasma as an adjunctive material to bone graft: a systematic review and metaanalysis of randomized controlled clinical trials. Int J Oral Maxillofac Surg. 2016 Aug; 45(8):1027-34

10 Badran KW, Sand JP. Platelet-Rich Plasma for Hair Loss: Review of Methods and Results. Facial Plast Surg Clin North Am. 2018 Nov; 26(4):469-85.

11 Bernardi S, Mummolo S, Tecco S, Continenza MA, Marzo G. Histological characterization of Sacco's concentrated growth factors membrane [Caracterización Histológica de la Membrana de los Factores de Crecimiento Concentrados Sacco]. Int J Morphol. 2017; 35(1):114-9.

12 Avila Rodríguez MI, Rodríguez Barroso LG, Sánchez ML. Collagen: A review on its sources and potential cosmetic applications. J Cosmet Dermatol. 2018 Feb;17(1): 20-6. 
13 Yang L, Zhang D, Wu H, Xie S, Zhang M, Zhang B, et al. Basic Fibroblast Growth Factor Influences Epidermal Homeostasis of Living Skin Equivalents through Affecting Fibroblast Phenotypes and Functions. Skin Pharmacol Physiol. 2018;31(5):229-37.

14 McCain J. The MAPK (ERK) Pathway: Investigational Combinations for the Treatment Of BRAF-Mutated Metastatic Melanoma. P T. 2013 Feb;38(2):96-108.

15 Choi EH. Changes of skin barrier with aging. In: Quan T. Molecular mechanisms of skin aging and age related diseases. Boca Raton: Taylor \& Francis Group, 2016. Cap 3. p. 6189 https://doi.org/10.1201/b21370-4.

16 Nikolakis G, et al. Agin Skin: A window to the dody. In: Quan T. Molecular mechanisms of skin aging and age related diseases. Boca Raton: Taylor \& Francis Group, 2016, cap 1.p. 200-227.

17 Roberts MS, Dancik Y, Prow TW, Thorling CA, Lin LL, Grice JE, et al. Non-invasive imaging of skin physiology and percutaneous penetration using fluorescence spectral and lifetime imaging with multiphoton and confocal microscopy. Eur J Pharm Biopharm. 2011 Apr;77(3):469-88.

18 Vieira AC, et al. Fatores de crescimento: uma nova abordagem cosmecêutica para o cuidado antienvelhecimento. Rev Bras Farm. 2011 92(3):80-9.

19 Zouboulis CC, Adjaye J, Akamatsu H, MoeBehrens G, Niemann C. Human skin stem cells and the ageing process. Exp Gerontol. 2008 Nov;43(11):986-97.

20 Fabi S, Sundaram H. The potential of topical and injectable growth factors and cytokines for skin rejuvenation. Facial Plast Surg. 2014 Apr;30(2):157-71.

21 Schwartz E, Cruickshank FA, Christensen CC, Perlish JS, Lebwohl M. Collagen alterations in chronically sun-damaged human skin. Photochem Photobiol. 1993 Dec;58(6): 841-4.

22 Quan T, Qin Z, Xia W, Shao Y, Voorhees JJ, Fisher GJ. Matrix-degrading metalloproteinases in photoaging. J Investig Dermatol Symp Proc. 2009 Aug;14(1):20-4.

23 Khavkin J, Ellis DA. Aging skin: histology, physiology, and pathology. Facial Plast Surg Clin North Am. 2011 May;19(2):229-34.

24 Zakrzewska M, Krowarsch D, Wiedlocha A, Otlewski J. Design of fully active FGF-1 variants with increased stability. Protein Eng Des Sel. 2004 Aug;17(8):603-11.

25 Baumann L. Skin ageing and its treatment. J Pathol. 2007 Jan;211(2):241-51.

26 Ruiz MA, Charles B, Morales ME, Gallardo V. Evaluation of the anti-wrinkle efficacy formulations with a anti-aging peptide
(Argireline $^{\circledR}$ ). Ars Pharm 2010;50(4):16876.

27 Zeka K, Ruparelia KC, Sansone C, Macchiarelli G, Continenza MA, Arroo RR. New Hydrogels Enriched with Antioxidants from Saffron Crocus Can Find Applications in Wound Treatment and/or Beautification. Skin Pharmacol Physiol. 2018;31(2):95-8.

28 Rozman P, Bolta Z. Use of platelet growth factors in treating wounds and soft-tissue injuries. Acta Dermatovenerol Alp Pannonica Adriat. 2007 Dec;16(4):156-65.

29 Fitzpatrick RE, Rostan EF. Reversal of photodamage with topical growth factors: a pilot study. J Cosmet Laser Ther. 2003 Apr;5(1): 25-34.

30 Palermo E, et al. Tratado de Cirurgia Dermatológica, Cosmiatria e Laser da Sociedade Brasileira de dermatologia. Rio de Janeiro: Elsevier; 2012.

31 Yamamoto Y, Gaynor RB. Therapeutic potential of inhibition of the NF-kappaB pathway in the treatment of inflammation and cancer. J Clin Invest. 2001 Jan;107(2):135-42.

32 Nelson DL. COX, Michael M. Lehninger principles of biochemistry. 6th ed. Basingstoke: Macmillan Education; 2013.

33 Żerańska J, Pasikowska M, Szczepanik B, Mlosek K, Malinowska S, Dębowska RM, et al. A study of the activity and effectiveness of recombinant fibroblast growth factor (Q40P/ S47I/H93G rFGF-1) in anti-aging treatment. Postepy Dermatol Alergol. 2016 Feb;33(1): 28-36.

34 Werner S. Keratinocyte growth factor: a unique player in epithelial repair processes. Cytokine Growth Factor Rev. 1998 Jun;9(2): 153-65.

35 Szwed DN, Santos VLP. Fatores De Crescimento Envolvidos Na Cicatrização De Pele. Caderno da Escola de Saúde, Curitiba. 2015; 1(15):7-17.

36 Yamasaki M, Miyake A, Tagashira S, Itoh N. Structure and expression of the rat mRNA encoding a novel member of the fibroblast growth factor family. J Biol Chem. 1996 Jul; 271(27):15918-21.

37 Werner S, Grose R. Regulation of wound healing by growth factors and cytokines. Physiol Rev. 2003 Jul;83(3):835-70.

38 Carneiro MC, et al. Growth factors in healing diabetic ulcers. Braz J Health Sci. 2013;11(38).

39 Yamaguchi TP, Rossant J. Fibroblast growth factors in mammalian development. Curr Opin Genet Dev. 1995 Aug;5(4):485-91.

40 Gospodarowicz D, Neufeld G, Schweigerer L. Molecular and biological characterization of fibroblast growth factor, an angiogenic factor which also controls the proliferation and differentiation of mesoderm and neuroecto- derm derived cells. Cell Differ. 1986 Jul;19(1): $1-17$.

41 Clarke MS, Khakee R, McNeil PL. Loss of cytoplasmic basic fibroblast growth factor from physiologically wounded myofibers of normal and dystrophic muscle. J Cell Sci. 1993 Sep;106(Pt 1):121-33.

42 Ornitz DM, Itoh N. The Fibroblast Growth Factor signaling pathway. Wiley Interdiscip Rev Dev Biol. 2015 May-Jun;4(3):215-66.

43 Wu M, Chen Y, Jiang L, Li Y, Lan T, Wang Y, et al. Type II cGMP-dependent protein kinase inhibits epidermal growth factor-induced phosphatidylinositol-3-kinase/Akt signal transduction in gastric cancer cells. Oncol Lett. 2013 Dec;6(6):1723-8.

44 Matsuo FS, et al. Estudo da via de sinalização PI3K-Akt E GSK3 $\beta$ em carcinomas epidermoides metastáticos e não metastáticos de cavidade bucal. Dissertation (Master degree). Federal University of Uberlândia, Minas Gerais, 2015

45 Carpenter G, Ji Q. Phospholipase C- $\gamma$ as a signal-transducing element. Exp Cell Res. 1999 Nov;253(1):15-24.

46 Kiu H, Nicholson SE. Biology and significance of the JAK/STAT signalling pathways. Growth Factors. 2012 Apr;30(2):88-106.

47 Hilling C. HGFs as natural counterparts. Cosmetics e Toiletries. 2010;22(5):44-7.

48 Xie L, Zhang M, Dong B, Guan M, Lu M, Huang $Z$, et al. Improved refractory wound healing with administration of acidic fibroblast growth factor in diabetic rats. Diabetes Res Clin Pract. 2011 Sep;93(3):396-403.

$49 \mathrm{Ha} \mathrm{JH}$, Kim HN, Moon KB, Jeon JH, Jung DH, Kim SJ, et al. Recombinant Human Acidic Fibroblast Growth Factor (aFGF) Expressed in Nicotiana benthamiana Potentially Inhibits Skin Photoaging. Planta Med. 2017 Jul; 83(10):862-9.

50 Artoni LP. Basic fibloblast growth factor and its receptors in relation to proliferative activity in the buffalo placenta during gestation. Arq Bras Med Vet Zootec. 2007;59(3):60513.

51 Rival D, Bonnet S, Sohm B, Perrier E. A Hibiscus Abelmoschus seed extract as a protective active ingredient to favour FGF-2 activity in skin. Int J Cosmet Sci. 2009 Dec;31(6):41926.

52 Ono I. A Study on the Alterations in Skin Viscoelasticity before and after an Intradermal Administration of Growth Factor. J Cutan Aesthet Surg. 2011 May;4(2):98-104.

53 Weiss RA, Weiss MA. Evaluation of a novel anti-aging topical formulation containing $\mathrm{cy}$ cloastragenol, growth factors, peptides, and antioxidants. J Drugs Dermatol. 2014 Sep; 13(9):1135-9. 